\title{
Pronoms indéfinis et grammaticalisation
}

quelques enseignements du japonais

\section{Makoto Kaneko}

\section{(2) OpenEdition Journals}

Édition électronique

URL : https://journals.openedition.org/ml/479

DOI : $10.4000 / \mathrm{ml} .479$

ISSN : 2274-0511

Éditeur

Association Modèles linguistiques

Édition imprimée

Date de publication : 1 juillet 2007

Pagination : 37-57

\section{Référence électronique}

Makoto Kaneko, «Pronoms indéfinis et grammaticalisation », Modèles linguistiques [En ligne], 56

2007, mis en ligne le 07 janvier 2014, consulté le 01 juillet 2021. URL : http://journals.openedition.org/ ml/479; DOI : https://doi.org/10.4000/ml.479 


\section{Pronoms indéfinis et grammaticalisation : quelques enseignements du japonais ${ }^{1}$}

Makoto Kaneko

\section{Introduction ${ }^{2}$}

Le japonais construit des expressions indéfinies et des questions partielles en associant un 'pronom indéterminé' (ex. dare, nani) avec une particule (ex. ka), comme en [1]-[4] $]^{3}$ :

\begin{tabular}{|c|c|c|}
\hline$[1] \mathrm{a}$ & $\begin{array}{l}\text { John-ga nani-ka-o } \quad \text { kat- ta. } \\
\text { John-Nom quoi-KA-Acc acheter-Pas }\end{array}$ & (Kuroda $1965: 97$ ) \\
\hline$[1] \mathrm{b}$ & \multicolumn{2}{|l|}{ John a acheté quelque chose. } \\
\hline$[2] \mathrm{a}$ & \begin{tabular}{llc} 
John-ga & nani- o & \multicolumn{1}{c}{ kat- ta- $[$ no-] ka. } \\
John-Nom & QUOI-Acc & acheter-Pas-[Comp]-KA
\end{tabular} & (idem. 98) \\
\hline$[2] \mathrm{b}$ & \multicolumn{2}{|l|}{ John a acheté quoi? } \\
\hline [3]a & $\begin{array}{lll}\text { dare-ka- ga } & \text { hon- o } & \text { kat- ta. } \\
\text { QUI-KA-Nom- } & \text { livre-Acc } & \text { acheter-Pas }\end{array}$ & (idem \\
\hline$[3] \mathrm{b}$ & \multicolumn{2}{|l|}{ Quelqu'un a acheté un livre. } \\
\hline$[4] \mathrm{a}$ & $\begin{array}{c}\text { kat- } \quad \text { ta-[no-] ka. } \\
\text { acheter-Pas-[Comp]-KA }\end{array}$ & \\
\hline $\mathrm{db}$ & Qui a acheté un livre? & \\
\hline
\end{tabular}

Le terme 'pronom indéterminé' (en anglais, 'indeterminate pronoun') est une appellation adoptée par Kuroda (1965). La linguistique traditionnelle japonaise parle de futei-go 'mot indéterminé' — « fu est un marqueur de négation et tei signifie 'décider, fixer' » (Blin 1994 : 63) alors que go veut dire 'mot'.

Sémantiquement, les pronoms indéterminés servent à introduire une

1 Je remercie Anne Zribi-Hertz et Laurent Roussarie pour leurs remarques précieuses et constructives. Toutes les insuffisances qui restent malgré leurs aides sont de mon fait.

2 Les abréviations utilisées dans cette étude sont les suivantes : $\mathrm{Abl}=$ ablatif ; $\mathrm{Acc}=$ accusatif ; $\mathrm{CL}=$ classificateur ; Comp $=$ complémenteur ; Cop = copule ; $\mathrm{CP}=$ syntagme de complémenteur ; Dat $=$ datif $; \mathrm{DM}=$ démonstratif $; \mathrm{Gen}=$ génitif $;$ Loc $=$ locatif $;$ Nom = nominatif $; \mathrm{SN}=$ syntagme nominal $; \mathrm{Num}=$ numéral $; \mathrm{Neg}=$ négation $;$ Prog $=$ progressif $;$ Pas $=$ passé $;$ Top $=$ topique $; \#=$ bien formé syntaxiquement, mais inapproprié dans son contexte discursif.

3 Dans tous les exemples japonais cités dans cette étude, les pronoms indéterminés et la particule $k a$ sont imprimés en gras. 
variable, mais ne disposent pas d'une force quantificationnelle propre : dare et nani fournissent respectivement une variable d'entité humaine et une variable d'entité non-humaine ou d'événement ${ }^{4}$. Dans cet article, je glose dare et nani par 'qui' et 'quoi', en suivant Kuroda (1965) qui recourt aux gloses par 'who' et 'what', bien que, comme le note Kuroda lui-même, ces gloses ne soient pas exactes, en ce que les pronoms indéterminés dare et nani sont dépourvus du sens interrogatif inhérent aux pronoms interrogatifs de l'anglais et du français. Il existe des pronoms indéterminés autres que dare et nani (ex. doko 'où', itu 'quand', etc.) et des particules autres que $k a^{5}$. Toutefois, la présente étude se concentrera sur l'association de dare et nani avec $k a^{6}$.

Si la particule $k a$ est attachée à dare ou nani, on obtient un effet de quantification existentielle (« indéfini positif » selon Chang \& Labrune 1994) : nani-ka en [1a] et dare-ka en [3] a sont traduisibles par 'quelque chose' en [1]b et par 'quelqu'un' en [3]b. D'autre part, la combinaison à distance du pronom et de $k a$ produit une question partielle : les associations nani... ka en [2]a et dare... $k a$ en [4a] correspondent aux pronoms interrogatifs qui en [2]b et quoi en [4]b.

Beaucoup de travaux antérieurs sont consacrés aux propriétés syntaxiques et sémantiques de la combinaison à distance illustrée en [2]a et $[4] \mathrm{a}^{7}$. En revanche, la combinaison adjacente illustrée en [1]a (nani-ka) et [3]a

4 Plus exactement, dans certains cas, le pronom indéterminé nani correspond à de l'humain : une question en nani permet une réponse dénotant un individu humain, comme en (Ia). De ce point de vue, nani est analogue au pronom interrogatif quoi en $(\mathrm{Ib})$, et doit être analysé comme sous-spécifié (non-catégorisé) pour le trait $[ \pm$ humain], comme le soutient Lefeuvre (2006) pour quoi :
(1)a A : nani- o mi- teiru- no- ka ? - B : ano onnanoko desu. QUOI-Acc regarder-Prog-Comp-KA DM fille Cop
(I)b A: Tu regardes quoi?
- B : Cette fille, là-bas.
(Lefeuvre 2006:39)

5 Par exemple, la particule mo construit un quantifieur universel, comme en (Ia) ou un item à polarité négative comme en $(1 \mathrm{~b})$, tandis que la particule demo forme un item de choix libre correspondant à « indéfini virtuel » chez Chang \& Labrune (1994) :

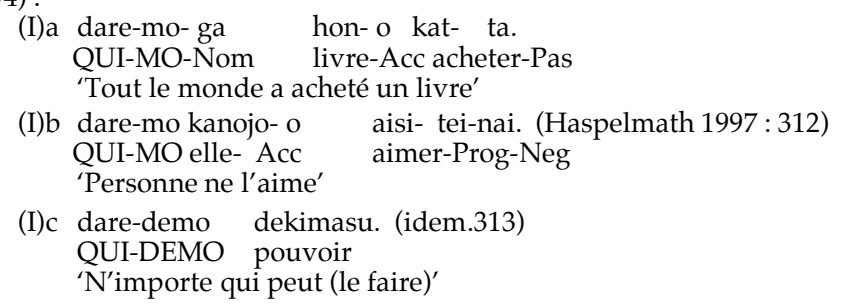

6 Pour les autres combinaisons d'un pronom indéterminé avec une particule, voir Shimoyama (2006: 143).

7 La combinaison à distance manifeste les contraintes distributionnelles suivantes : $k a$ dans la matrice peut être combinée avec un pronom indéterminé qui se situe à l'intérieur d'une relative comme en (I) ; plusieurs pronoms indéterminés peuvent être associés à une seule particule $k a$, mais l'association doit être locale et ne peut pas être interrompu par une autre particule, comme en (II) où la présence de $k a$ dans la subordonnée empêche l'association de dare et nani dans la subordonnée 
[dare-ka] a été moins étudiée : il est généralement admis que ces deux expressions indéfinies sont des pronoms correspondant à quelque chose et quelqu'un en français, et que $k a$ produit l'effet d'indéfinitude comme un déterminant fournissant le quantifieur existentiel (cf. Watanabe 2006). Selon cette analyse, le $k a$ d'indéfinitude est radicalement différent du $k a$ d'interrogation.

Je me propose tout d'abord de montrer que nani-ka et dare-ka donnent lieu à des emplois autres que pronominaux (par ex. adverbial) et à deux expressions dérivées (une particule de focus et une particule de discours). Afin de relier entre eux ces différents emplois, j'aurai recours à la notion de grammaticalisation, définie par Meillet (1958: 131) comme « le passage d'un mot autonome au rôle d'élément grammatical » entraînant la contraction phonologique, l'intégration morphologique, la réanalyse (la restructuration) syntaxique, et / ou l'extention sémantique. Je chercherai ensuite à relier l'une à l'autre la combinaison adjacente dare-ka (cf.[3]a) et la combinaison à distance dare...ka (cf. [4]a), que je proposerai de rapprocher, respectivement, du pronom indéfini complexe je ne sais qui (cf. [5a]), et de la séquence je ne sais pas qui (cf [5]b), qui introduit une question enchâssée :

[5]a Je ne sais qui a acheté un livre. (pronom indéfini complexe)

[5]b Je ne sais pas qui a acheté un livre. (question enchâssée)

Dans ce qui suit, je présenterai d'abord certains travaux antérieurs pertinents pour la discussion ( $\S 2$ ). J'aborderai ensuite les emplois non nominaux de nani-ka et dare-ka, et discuterai de manière critique l'hypothèse de Mikami (1972) selon laquelle l'emploi premier est adverbial, en examinant l'emploi dérivé de nani-ka (particule de focus) (§3). Puis, je proposerai pour dare-ka et nani-ka une structure interne complexe analogue à une question enchâssée, et tenterai de rendre compte de leur divers emplois en termes de grammaticalisation pour mieux comprendre la notion d'indéfinitude (§ 4). La section 5 récapitulera les résultats.

\section{Travaux antérieurs}

Dans cette section, je présenterai d'abord la liste des interprétations possibles de dare-ka et nani-ka, proposée par Haspelmath (1997) (§ 2.1). J'envisagerai ensuite une observation de Nishigauchi (1990), qui montre que la position de la particule $k a$ permet de leur enlever toute ambiguïté (§ 2.2). J'aborderai enfin certains travaux qui tentent de préciser la fonction essentielle de $k a(\S 2.3)$.

avec un autre $k a$ dans la principale. Du coup, cet exemple ne donne qu'une interrogation polaire et non une interrogation partielle, à la différence de (I) :

(I) Taro-wa ([dare-ga kat- ta] moti)- o tabemasi-ta-ka ? Taro-Top [[QUI-Nom acheter-Pas] gâteau de riz]-Acc manger-Pas-KA

'Lit. Qui ${ }^{k}$-est tel que Taro a mangé un gâteau de riz qu ${ }^{k}$ 'il avait acheté ?'

'Qui a acheté le gâteau de riz mangé par Taro ?' (Shimoyama 2006 : 142)

(II) Taro-wa [Yamada-ga dare-ni nani-o okut- ta ka] tazunemasi-ta-ka ? T.- Top Y.- Nom QUI-Dat QUOI-Acc envoyer-Pas-KA demander-Pas-KA 'Taro a-t-il demandé à qui Yamada avait envoyé quoi ?' (idem.141)

Pour les explications de ces faits du point de vue syntaxique ou sémantique, voir notamment Nishigauchi (1990), Watanabe (1992), Shimoyama (2006). 


\subsection{Trois interprétations de dare-ka et nani-ka}

Selon Nihon-kokugo-daijiten (Grand dictionnaire de la langue japonaise 2003 : 209), nani-ka sert

1) à dénoter une entité ou un événement qui ne sont pas clairement identifiés, mais qui existent réellement,

2) à éviter de donner l'identité précise d'une entité ou d'un événement qui existent réellement, ou

3) à dénoter une entité ou un événement qui ne sont que susceptibles $\mathrm{d}$ 'exister. Ces trois emplois sont également constatés pour dare-ka.

Haspelmath (1997) reformule cette description tri-partite dans les termes suivants :

1) indéfini spécifique inconnu du locuteur (specific unknown to the speaker) (désormais : -FAM, càd, 'non familier au locuteur'), illustré par [6]a ;

2) indéfini spécifique connu du locuteur (specific known to the speaker) (désormais : +FAM), comme en [6]b ;

3) indéfini non spécifique, cas observé dans une phrase à modalité irréelle comme [6]c ou incluant un adverbe quantificationnel (ex. toujours) comme [6]d :

[6]a dare-ka kara denwa at- ta kedo, dare-kara-da- ka wakara-nai. QUI-KA Abl téléphone il y a-Pas mais Qui-Abl-Cop-KA savoir-Neg 'Il y a un appel de quelqu'un, mais je ne sais pas de qui'

(Haspelmath 1997 : 314)

[6]b dare-ka kara denwa at- ta kedo, dare-kara-da-ka ate- tegoran. QUI-KA Abl téléphone il y a-Pas mais QUI-Abl-Cop-KA deviner-Impératif 'Il y a un appel de quelqu'un, et devine de qui !' (ibid.)

[6]c dare-ka ni kii- temimas-yoo. (ibid.)

Qui-KA Dat demander-tenter-je vais 'Je vais questionner quelqu'un.'

[6]d itumo dare-ka kara denwa- ga aru. Toujours QUI-KA Abl téléphone-Nom il y a

'Il y a toujours un appel de quelqu'un'

La relation entre ces trois interprétations est schématisée par l'échelle d'indéfinitude en [7] : plus on est à gauche sur l'échelle, plus on a un référent individualisé et saillant pour le locuteur.

[7] échelle d'indéfinitude

$$
\begin{array}{ll}
\text { spécifique connu } \rightarrow \text { spécifique inconnu } \rightarrow \text { non-spécifique } \\
{[6] \mathrm{b}[+\mathrm{FAM}]} & {[6] \mathrm{a}[-\mathrm{FAM}]} \\
{[6] \mathrm{c}, \mathrm{d}}
\end{array}
$$

\subsection{Position de $k a$ par rapport à la marque casuelle}

Nishigauchi (1990) fait remarquer que la position de $k a$ par rapport au marqueur casuel a pour effet de supprimer l'ambiguïté : dans les exemples suivants, le prédicat todoita 'est arrivé' dénotant un événement réel spatiotemporellement délimité, force la lecture spécifique. La suite $<$ dare-ka-Cas $>$ en [8]a permet soit une lecture spécifique (+FAM), soit une lecture spécifique 
$(-\mathrm{FAM})^{8}$, alors que la suite $<$ dare-Cas-ka> en [8]b n'admet que la seconde lecture :

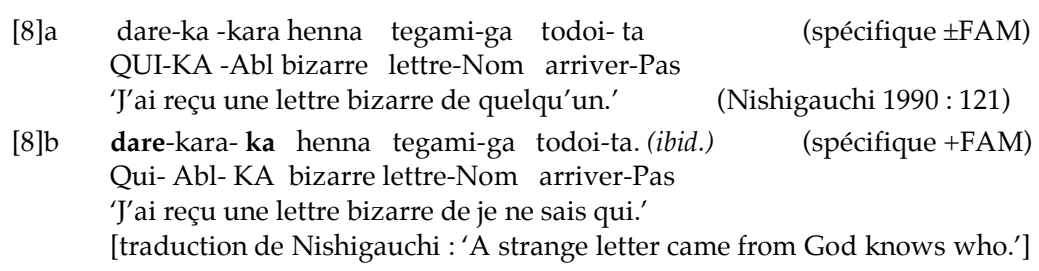

Shimoyama (2006 : 144) soutient que la particule $k a$ qui suit le cas ablatif en [8]b marque l'interrogation ${ }^{9}$ et que la possibilité d'intercaler la copule $d a$ entre <dare-CAS> et $k a$ suggère qu'il s'agit de la forme elliptique d'une interrogative enchâssé $e^{10}$, paraphrasable par je ne sais qui. Je postulerai plus loin que cette analyse en termes d'interrogation enchâssée s'applique également à la source étymologique de la combinaison adjacente dare-ka.

\subsection{Principales fonctions de la particule $k a$}

La particule $k a$ sert également à marquer la disjonction, comme en [9]a,b. Kuroda (1965), Tamba-Mecz (1984), Okutsu (1996), entre autres, mettent en relief la relation étroite entre le $k a$ d'interrogation et le $k a$ de disjonction : selon Tamba-Mecz, « ces deux particules spécifient que la relation sur laquellle elles portent n'est qu'une possibilité parmi d'autres » (p. 227). Kuroda préconise, en outre, d'une part un rapprochement entre le $k a$ de disjonction propositionnelle comme en [9]b et le $k a$ d'interrogation comme en [1]b, et $\mathrm{d}^{\prime}$ autre part un rapprochement entre le $k a$ de disjonction nominale comme en [9]a et le $k a$ d'indéfinitude comme en [1]a. Okutsu (1996) cite en effet l'exemple [9]c où dare-ka est précédé de SN disjoints comme en [9]c :

[9]a John-ka Bill-ka-ga hon-o kat- ta. (Kuroda $1965: 85$ )

John-KA Bill-KA-Nom livre-Acc acheter-Pas

'C'est soit John, soit Bill qui a acheté un livre.' (disjonction nominale)

9]b John-ga hon-o kat- ta- ka Bill-ga hon-o kat- ta-ka desu John-Nom livre-Acc acheter-Pas-KA Bill-Nom livre-Acc acheter-Pas-KA-Cop 'C'est soit John qui a acheté un livre, soit Bill qui a acheté un livre.' (idem. 86) (disjonction propositionnelle)

[9]c watasi-ka anata-ka soretomo kare-ka, dare-ka wa rusubansi-nakutewanaranai. moi-KA toi-KA ou bien lui-KA, QUI-KA Top surveiller la maison-devoir 'Quelqu'un comme moi, toi, ou bien lui doit surveiller la maison.'

(Okutsu 1996 : 159)

(disjonction nominale)

8 Nishigauchi lui-même traite de ce contraste interprétatif en termes de la distinction spécifique / non-spécifique. Toutefois, dans l'optique de Haspelmath, c'est la distinction spécifique $( \pm F A M)$ qui est pertinente.

9 Voir aussi Nishigauchi (1990 : 121) qui suggère que sémantiquement, "la séquence $<$ dare-Cas-ka $>$ se situe à mi-chemin entre un SN indéfini et une question enchâssée 'The semantics of PP [Post-positional Phrase équivalent aux marques casuelles]-ka is somewhere in between an indefinite SN and an embedded question' ».

10 " The particle $k a$ in this example could possibly be the question marker $k a[\ldots]$ darekara-ka is interchangeable with dare-kara-da-ka 'from whom-Cop-KA', which suggests that it might very well be an elliptical form of embedded question» (Shimoyama $2006: 144$ ) 
D'autre part, observant que l'indéfinitude correspond à la quantification existentielle qui se réduit logiquement à la disjonction généralisée (i.e. $\exists x P(x)=P(a) \vee P(b) \vee P(c) \vee$...) mais que typologiquement, une forme identique marque rarement à la fois la disjonction et l'indéfinitude, Haspelmath (1997 : 164-169) suggère que la relation entre ces deux fonctions n'est qu'une «coïncidence» et avance l'hypothèse qu'elles sont diachroniquement dérivées d'une même source.

En ce qui concerne la relation entre le $k a$ d'interrogation et le $k a$ d'indéfinitude, la plupart des travaux antérieurs s'accordent à les distinguer, quoique Nishigauchi (1990) suggère l'existence d'un cas intermédiaire (voir la note 9). Parmi ceux qui cherchent une explication unifiée, Hagstrom (1998: 25-26) attire l'attention sur le fait que dans les questions partielles de l'ancien japonais, dare ou nani et $k a$ sont directement attachés, cependant que le prédicat verbal prend une forme spéciale : en ancien japonais, le prédicat prend normalement la forme conclusive en proposition principale, tandis qu'en proposition relative ou nominalisée, le prédicat prend une forme appelée 'adnominale', comme en [10]a :

[10]a tare ${ }^{11}$ - ka mata hanatatibana-ni omoi-idemu. QUI- KA encore fleur d'orange-Dat se souvenir adnominale $_{\text {a }}$

'(Je me demande) qui se souviendra de moi à (l'odeur de) la fleur d'oranger ?'[Shin-Kokin-Wakashuu (au début de $13^{\mathrm{ème}}$ ) cité par Hagstrom $1998: 25]$

[10]b dare-ka...prédicat ${ }_{\text {forme adnominale }} \rightarrow$ dare...prédicat- $k a$ [évolution diachronique]

$[10] \mathrm{c} \quad$ dare-ka...prédicat $\left[{ }_{\text {comp }} \mathrm{Q}\right] \rightarrow$ dare...prédicat $-\left[{ }_{\text {comp }} \mathrm{Q}+\boldsymbol{k a}\right.$ ] [dérivation en japonais moderne]

Dans la linguistique traditionnelle japonaise, cette corrélation entre la particule (appelée kakari) et une forme particulière de conjugaison (appelée musubi) est appelée kakari-musubi (corrélat-conclusion). En japonais moderne, la distinction entre formes adnominale et conclusive est neutralisée et les phénomènes de kakari-musubi ont disparu. Corrélativement, pour préciser qu'il s'agit d'une question, le ka est attaché au prédicat, comme le schématise [10]b.

Hagstrom avance l'hypothèse que la dérivation syntaxique du japonais moderne reflète ce changement diachronique : dans la première étape de la dérivation, la particule $k a$ est adjacente à dare et la tête du complémenteur est dotée d'un trait de question « $Q$ » qui est responsable de la forme adnominale en ancien japonais ; la position de surface est dérivée par le mouvement de $k a$ jusqu'à la tête Complémenteur, comme en [10]c. D'après Hagstrom, la particule $k a$ est, sémantiquement, un quantificateur existentiel portant sur une fonction de choix ${ }^{12}$ qui « s'applique à un ensemble et en extrait un élément

11 Le mot tare en ancien japonais correspond au pronom indéterminé dare 'qui' en japonais moderne.

12 Yatsushiro (1996) soutient d'autre part que ka ne sert qu'à introduire une fonction de choix. 
particulier» (Dobrovie-Sorin \& Beyssade $2004: 19)^{13}$. Selon cet auteur, le $k a$ d'indéfinitude est donc la source du $k a$ d'interrogation. Dans ce qui suit, je soutiendrai à l'inverse que le $k a$ d'interrogation est la source du $k a$ d'indéfinitude.

\section{Emplois non pronominaux de dare-ka et nani-ka}

Dans cette section, je présenterai d'abord l'hypothèse de Mikami (1972) selon laquelle dare- $k a$ et nani-ka sont essentiellement de nature non-nominale et que leur emploi nominal résulte de la réanalyse syntaxique (§ 3.1). Je discuterai ensuite cette hypothèse de manière critique, en examinant la distribution de la particule de focus nanka (§3.2).

\section{1. dare-ka et nani-ka en position non argumentale}

Mikami (1972), Kamio (1973) (entre autres) observent des comportements parallèles entre dare-ka / nani-ka et la suite <NUMERAL+CLASSIFICATEUR > : en [11]a, dare-ka se situe à l'extérieur du sujet (souligné) marqué pour le nominatif et peut être analysé comme adverbial ; en l'absence du SN, dare-ka peut être ou ne pas être marqué pour le nominatif, comme en [11]b,c. Les mêmes distributions s'observent avec la suite futa-ri 'deux-CL' (en gras), en [12]a,b,c :

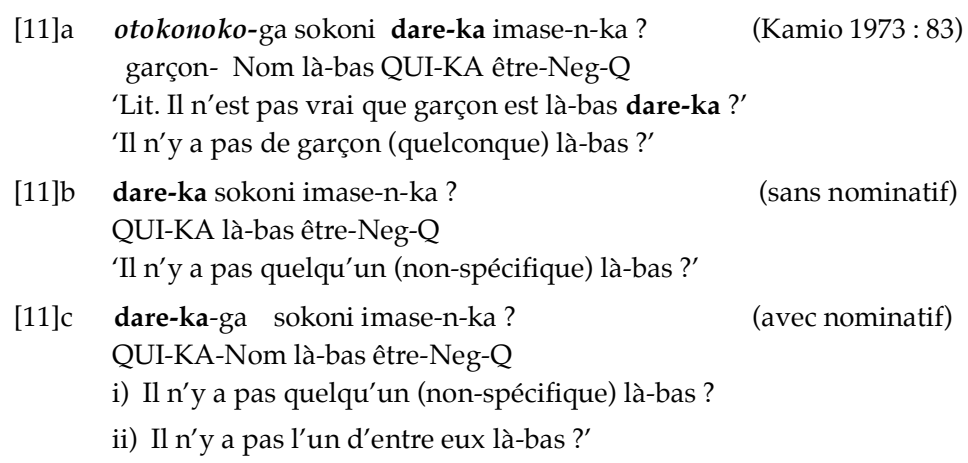

[12]a otokonoko-ga sokoni futa-ri imase-n-ka? garçon- Nom là-bas deux-CL être-Neg-Q 'Lit. Il n'est pas vrai que garçon $_{\mathrm{Nom}}$ est là-bas deux personnes ?' 'Il n'y a pas deux garçons (quelconques) là-bas ?'

[12]b futa-ri sokoni imase-n-ka? (sans nominatif) deux-CL là-bas être-Neg-Q 'Il n'y a pas deux personnes (quelconques) là-bas ?'

[12]c futa-ri-ga sokoni imase-n-ka? (avec nominatif) QUI-KA-Nom là-bas être-Neg-Q

i) '`Il n'y a pas deux personnes (quelconques) là-bas ?'

ii) 'Les deux personnes ne sont pas là-bas.'

13 En termes plus techniques, une fonction de choix «s'applique à la propriété dénotée par le prédicat nominal et retourne comme valeur un individu quelconque, qui a la propriété en question ». 
Mikami soutient par ailleurs que dare-ka et futa-ri ont les mêmes propriétés interprétatives ;

4) la suite futa-ri est interprétée comme définie avec une marque casuelle en [12]c, et comme indéfinie non spécifique dans une position adverbiale en [12]a. Si le cas est absent en [12]b, la lecture définie est difficile à obtenir ;

5) dare-ka n'admet que l'interprétation indéfinie non spécifique en position adverbiale en [11]a, et permet l'interprétation partitive avec le cas, comme le suggère la traduction française de $[11] \mathrm{c}^{14}$. Dans l'optique d'Enç (1991) qui argue que l'indéfini spécifique se réduit à l'interprétation partitive, dare-ka casuellement marqué produit l'interprétation indéfinie spécifique. Corrélativement, si le cas est absent comme en [11]b, la lecture partitive (spécifique) est difficile à obtenir.

Les parallélismes formel et interprétatif que Mikami suggère entre les suites $<\mathrm{Num}-\mathrm{CL}>$ et $<$ dare-ka $>$ sont récapitulés en [13] sous forme d'échelle d'indéfinitude. Cet auteur suggère en outre que dans les deux cas, un élément syntaxiquement détaché [adverbial ou adjoint] est de plus en plus intégré dans la structure argumentale : $<\mathrm{NUM}-\mathrm{CL}>$ et $<$ dare-ka $>$ qui sont à l'origine de nature adverbiale acquerraient un statut nominal grâce au marquage casuel en passant par l'étape où la suite $<\mathrm{SN}+\mathrm{CAS}>$ est omise. La direction de la réanalyse syntaxique est signalée en [13] par les flèches :

[13] Réanalyses uni-directionnelles de Mikami (1972)

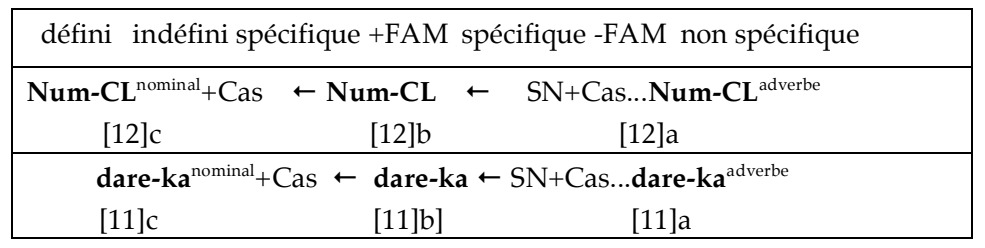

L'hypothèse de Mikami soulève toutefois au moins deux problèmes. D'abord, comme le fait remarquer Kawaguchi (1982 : 184) et comme l'indique le tableau [13], le parallélisme interprétatif n'est pas complet : la séquence $<$ Num-CL+CAS $>$ s'interprète obligatoirement comme définie, tandis que la suite $<$ dare $-k a+$ Cas $>$ ne permet jamais cette interprétation.

En second lieu, comme le note Mikami (1972) lui-même, les suites dare-ka et $<\mathrm{NUM}+\mathrm{CL}>$ peuvent précéder $<\mathrm{SN}+\mathrm{Cas}>$ comme en [14]a,b. Kamio (1973)

14 Dans la même veine, Hagstrom (1998) rapporte une observation due à Shigeru Miyagawa et à Mamoru Saito selon laquelle nani-ka marqué par l'accusatif ne permet pas, comme le montre (I), la reprise au moyen d'un pronom 'âne' (donkey pronoun), qui est permise en l'absence de l'accusatif. Hagstrom (1998 : 132) note que, selon Saito, cela suggère $\mathrm{qu}^{\prime}$ « un marquage casuel pourrait à sa façon donner la spécificité 'a case marking might in some way confer specificity " :

(I) MIT Press-ga $\left\{{ }^{*}\left[\right.\right.$ nani- ka]k-o/ $\left.{ }^{\mathrm{ok}}[\text { nani- } k a]_{k}\right\}$ syuppansur-eba, John-ga MIT Press-Nom \{QUOI-KA-Acc / QUOI-KA\} publier-Condition John-Nom taitei sorek-o yomu en général DM-Acc lire. (Hagstrom 1998: 132, ex. dû à Shigeru Miyagawa)

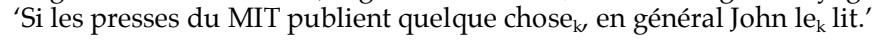


met toutefois en évidence que dare-ka prénominal comme en [14a] manifeste une distribution syntaxique analogue à celle de la suite $<\mathrm{NUM}+\mathrm{CL}+\mathrm{GEN}>$ comme en [14]c plutôt qu'à celle de la suite $<\mathrm{NUM}+\mathrm{CL}>$ prénominale sans génitif comme en [14]b :

[14] a dare-ka otokonoko-ga sokoni imase-n-ka? QUI-KA garçon-Nom là-bas être-Neg-Q

(Kamio $1973: 83$ ) 'Il n'y a pas de garçon [quelconque] là-bas ?

[14]b futa-ri otokonoko-ga sokoni imase-n-ka ? deux-CL garçon-Nom là-bas être-Neg-Q 'Il n'y a pas deux garçons là-bas ?'

[14]c futa-ri-no otokonoko-ga sokoni imase-n-ka ? deux-CL-Gen garçon-Nom là-bas être-Neg-Q 'Il n'y a pas deux garçons là-bas ?'

D'abord, la séquence $<$ dare- $k a+\mathrm{SN}>$ peut être coordonnée avec un autre $\mathrm{SN}$ comme en [15]a. Une telle coordination est possible pour $<\mathrm{Num-CL}-$ Gen $+\mathrm{SN}>$ comme en [15]b, et non pour $<\mathrm{Num}-\mathrm{CL}+\mathrm{SN}>$ en [15]c :

[15] a (ano onnanoko-to dare-ka otokonoko)-ga inakunat- ta- sooda. (ibid.) (cette fille et QUI-KA garçon)- Nom disparaître-Pas-on dit 'On dit que cette fille et je ne sais quel garçon ont disparu.'

[15]b (ano onnanoko-to futa-ri-no otokonoko)-ga inakunat- ta- sooda. (cette fille et deux-CL-Gen garçon)-Nom disparaître-Pas-on dit 'On dit que cette fille et deux garçons ont disparu.'

[15]c *(ano onnanoko-to futa-ri otokonoko)-ga inakunat- ta- sooda. (cette fille et deux-CL garçon)-Nom disparaître-Pas-on dit

Deuxièmement, $<\mathrm{SN}-\mathrm{Cas}+$ dare- $k a>$ connaît une restriction de cas : cette séquence n'est possible qu'au nominatif comme en [11]a ou à l'accusatif. Ainsi, la séquence $<$ dare- $k a+\mathrm{SN}-\mathrm{Abl}$ ] $>$ est parfaitement acceptable en [16]a, tandis que $<\mathrm{SN}-\mathrm{Abl}+$ dare-ka $>$ n'est pas acceptée en [16]b :

[16]a dare-ka gakusee-kara dengon- ga aru-yoodesu. (idem. 84) QUI-KA étudiant-Abl message-Nom être-sembler 'Il semble y avoir un message de je ne sais quel étudiant.'

[16]b *gakusee-kara dare-ka dengon-ga aru-yoo-desu. (ibid.) étudiant-Abl QUI-KA message-Nom être-sembler

$<$ Num-CL-Gen+SN-Abl $>$ est possible comme en [17]a, tandis que $<$ Num$\mathrm{CL}+\mathrm{SN}-\mathrm{Abl}>$ n'est pas acceptable comme en [17]b non plus que $<\mathrm{SN}$ $\mathrm{Abl}+\mathrm{Num}-\mathrm{CL}>$ comme en [17]c :

[17]a futa- ri- no gakusee-kara tegami-ga todoi-ta. (idem. 79) deux-CL Gen étudiant-Abl lettre-Nom arriver-Pas 'J'ai reçu des lettres de deux étudiant.'

[17]b *futa- ri gakusee-kara tegami- ga todoi-ta. deux-CL étudiant-Abl lettre-Nom arriver-Pas

[17]c *gakusee-kara futa- ri tegami-ga todoi- ta. étudiant-Abl deux-CL lettre-Nom arriver-Pas 
En se basant sur ces données, Kamio soutient que la suite $<\mathrm{Num-CL}>$ prénominale se situe à l'extérieur $\mathrm{du} \mathrm{SN}$, tandis que le dare-ka prénominal est interne au $\mathrm{SN}^{15}$. Ces différences syntaxiques indiquent que dare-ka ne peut pas être analysé comme un adverbial, parallèlement à la suite $<\mathrm{Num-CL}>$.

Dans la prochaine section, je présenterai d'autres faits qui jettent un doute sur l'hypothèse de Mikami selon laquelle nani-ka est, à l'origine, adverbial.

\subsection{La particule de focus nanka}

Nanka, forme contractée de nani-ka, sert de particule de focus ${ }^{16}$. La contraction est facultativement observée pour le pronom indéfini nani-ka, notamment dans la langue parlée, mais elle est obligatoire pour la particule de focus même dans le registre écrit.

Les particules de focus sont illustrées en français par des expressions comme aussi et seul (cf. [18]a,b). Le focus est ici défini, suivant Rooth (1996), comme mettant en oeuvre un ou des termes contrasté(s) avec le focus, appelé(s) 'Alternative Set' en anglais. La fonction sémantique des particules de focus est d'introduire ce(s) terme(s) contrasté(s) en spécifiant la relation qui unit le focus et ses termes contrastés au reste de la proposition; la particule additive aussi en [18]a asserte que le focus Jean est venu, alors que la particule exclusive seul en [18]b le présuppose. En outre, aussi présuppose l'existence $\mathrm{d}^{\prime}(\mathrm{au}$ moins) un terme contrasté qui est venu, tandis que seul en asserte l'absence :

[18]a Jean aussi est venu.

[18]b Seul Jean est venu

La particule nanka est placée à droite du focus et permet au moins les deux emplois illustrés en [19] : en [19]a, elle indique que le focus Taro incarne un exemple parmi ceux qui sont venus (emploi d'exemplification); en [19]b, elle sert à renforcer le jugement négatif du locuteur concernant l'existence d'un animal sans odeur (emploi d'évaluation négative) :

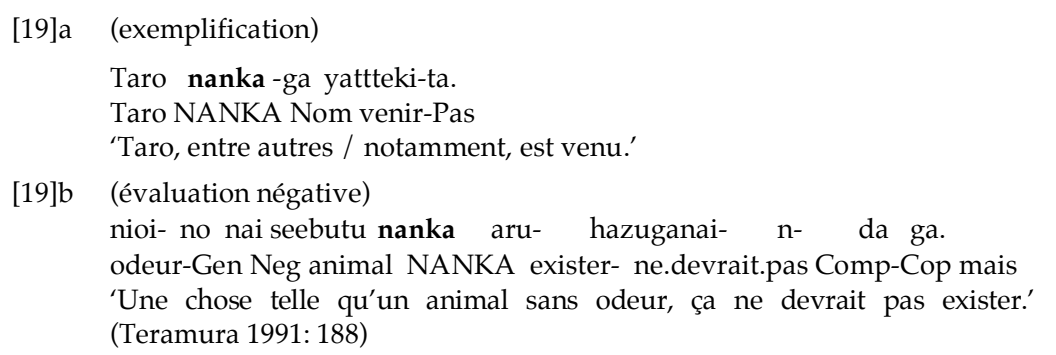
La fonction de la particule nanka sera mieux comprise en la comparant avec the like en anglais, illustré en [20]a,b :

15 On peut rapprocher la séquence <dare-ka / nani-ka+SN> de <quelque chose de+adjectif $>$ ou de <je ne sais qui /je ne sais quoi de+adjectif $>$ en français (ex. quelque chose de choquant, je ne sais quoi d'infâme (Lefeuvre 2006 : 162)). Willems (1998 : 142) suggère que le «statut syntaxique [de quelque chose] est proche du déterminant ».

16 Les linguistes japonais (Teramura 1991, Okutsu 1996, entre autres) l'appellent toritate-si qui signifie 'expression qui sert à mettre en relief'. 
[20]a [exemplification]

Taro and the like came.

'Taro et compagnie sont venus.'

[20]b [évaluation négative]

There shouldn't exist the like of an animal without smell.

'Une chose telle qu'un animal sans odeur, ça ne devrait pas exister.'

Sémantiquement, nanka et the like introduisent, sous la forme d'indéfini, l'ensemble des termes contrastés avec le focus, et sont paraphrasés par « quelqu'un / quelque chose qui appartient à cet ensemble » ${ }^{17}$.

Martin (1987) fait remarquer que les deux emplois de la particule nanka sont également exprimés par le nanka précédé, comme the like en [20]a, de la marque conjonctive ya, soulignée en [21]a,b. Étant donné que la conjonction met en relation des expressions de même catégorie syntaxique, nanka est analysé comme un SN en [21]a,b. Cet emploi de nanka est proche des cas où nani-ka ou dare-ka est précédé d'un SN, illustrés en [22]a,b :

[21]a Taro ya nanka- ga yattteki-ta.

Taro et NANKA- Nom venir-Pas

'Taro et son compagnie sont venus.'

[21]b nioi- no nai seebutu ya nanka aru- hazuganai- $n-$ da ga. odeur-Gen Neg animal et NANKA exister-ne.devrait.pas Comp-Cop mais 'Une chose telle qu'un animal sans odeur, ça ne devrait pas exister.'

[22]a ninjin ya tamanegi ya nani-ka- o irete... (adapté d'Okutsu $1996: 162$ ) carotte et oignon et QUOI-KA-Acc mettre...

'On y met quelque chose comme de la carotte, ou de l'oignon.'

[22]b watasi-ka anata-ka soretomo kare-ka, dare-ka wa rusubansimoi-KA toi-KA ou bien lui-KA, QUI-KA Top surveiller la maisonnakutewanaranai.

devoir

'Quelqu'un comme moi, toi, ou bien lui, doit surveiller la maison.' (=[9]c)

[22]c dare-ka ga rusubansi- nakutewanaranai.

QUI-KA Nom surveiller la maison-devoir

'Quelqu'un doit surveiller la maison.' (=[9c])

Les constructions telles que [22]a,b sont soigneusement étudiées par Okutsu (1996). Selon cet auteur, l'emploi d'un pronom indéfini présuppose souvent, comme dans l'interprétation partitive d'un indéfini, un ensemble de candidats contextuellement restreints, ce qui est analogue à l'ensemble des termes contrastés mis en oeuvre par le focus. Par exemple, dare-ka en [22c] sélectionne quelqu'un parmi ceux qui peuvent surveiller la maison en question à un moment donné.

17 Une analyse analogue est proposée par Siegel (2002) pour la particule de discours like (voir § 4.1). Du point de vue sémantique, l'introduction des termes contrastés se réduit à l'élargissement du domaine (Kadmon \& Landman (1993) sont les premiers à discuter cette notion sur la base de l'ambiguitté de any en anglais - choix libre ou item à polarité négative. D'après Kratzer \& Shimoyama (2002 : 14-15), l'élargissement du domaine a trois motivations pragmatiques : (a) éviter les assertions fausses ; (b) éviter les fausses inférences d'exhaustivité ; (c) renforcer l'assertion. L'emploi d'exemplification et l'emploi d'évaluation négative peuvent être corrélés respectivement aux motivations (b) et (c), tandis que la motivation (a) est instanciée par la particule de discours discutée en $\S 4.1$. 
En japonais, cette restriction contextuelle peut être effectuée extensionnellement par l'énumération [souvent non exhaustive] des candidats, comme en $[22] \mathrm{a}, \mathrm{b}^{18}$. Selon Okutsu, cette énumération peut inclure soit le conjonctif ya, comme en [22]a, soit le disjonctif $k a$, comme en [22]b : dans les deux cas, la partie qui précède le pronom indéfini ne sert qu'à introduire d'autres candidats à la sélection. Qu'ils soit superficiellement disjoints ou conjoints, la relation entre les divers candidats est déterminée par le disjonctif ka dans nani-ka ou dare-ka, comme cela est schématisé en [23]a,b :

$\begin{array}{lll}{[23] \mathrm{a}} & \{\text { carotte, oignon, } . .\} \text { nani-ka }[\vee]=\{\text { carotte } \vee \text { oignon } \vee \ldots\} & \text { (pour [22]a) } \\ {[23] \mathrm{b}} & \{\text { moi, toi, lui,... }\} \quad \text { dare-ka }[\mathrm{V}]=\{\text { moiVtoiVlui } \vee . .\} \quad \text { (pour [22]b) }\end{array}$

La restriction contextuelle des candidats présupposés peut aussi être effectuée intensionnellement comme en [24]a. Dans ce cas, dare-ka ou nani-ka peut occuper la position prénominale à l'intérieur du SN (voir la discussion de §3.1), comme en [24]b :

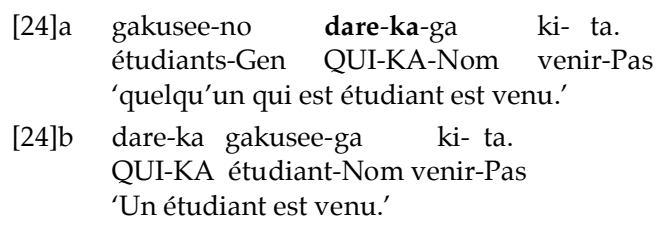

En [24]a, dare-ka est marqué pour le cas et peut donc être analysé comme le SN noyau, tandis qu'en [24]b, c'est le SN gakusee 'étudiant' qui est marqué casuellement et peut être analysé comme le SN noyau, auquel dare-ka est apposé. Compte tenu de cette observation, Okutsu suggère que dans le SN complexe (ex. tamanegi ya nani-ka 'quelque chose comme de l'oignon') formé de la partie dénotant partiellement l'ensemble des candidats présupposés (ex. tamanegi ya) et du pronom indéfini (ex. nani-ka), chacune des deux parties peut a priori être analysée soit comme le SN noyau, soit comme le SN apposé.

L'analyse syntaxique comme un SN appposé est plus évidente lorsque nani-ka est morphologiquement contracté et sémantiquement sous-spécifié pour pouvoir être combiné avec Taro-ya 'Taro et' dont le trait est (+humain), comme en [21]a. De ce point de vue, la particule de focus nanka est une forme plus grammaticalisée du SN apposé nanka, comme cela est schématisé en [25]a,b :

[25] SN noyau ou apposé SN apposé particule

[25]a tamanegi ya nani-ka $\rightarrow$ tamanegi ya nanka $\rightarrow \quad$ tamanegi nanka oignon et QUOI-KA oignon et NANKA oignon NANKA 'quelque chose comme de l'oignon' 'de l'oignon, entre autres,'

[25]b Taro ya Ziro ya dare-ka $\rightarrow$ Taro ya $\left\{\right.$ nanka $/{ }^{*}$ dare-ka $\rightarrow \rightarrow$ Taro $\left\{\right.$ nanka $/{ }^{*}$ dare-ka $\}$ Taro et Ziro et QUI-KA Taro $\{$ NANKA /QUI-KA 'quelqu'un comme Taro et Ziro' 'Taro, entre autres'

18 Eguchi (1998) fait remarquer que cette construction formée du SN appositif dénotant la restriction contextuelle et du SN noyau n'implique pas nécessairement un pronom indéfini, en citant des exemples comme (I) :

(I) Saeko-ya Mitsutosi-ya hadena yatu (Eguchi 1998 : 9)

Saeko et Mitsutosi et tapageur type

'les types tapageurs comme Saeko et Mitsutoshi' 
Envisageons maintenant les deux emplois de la particule nanka dans l'optique de la distinction entre indéfini spécifique et non spécifique:

6) Taro [ya] nanka (exemplification) indique qu'un individu contrasté avec Taro est effectivement venu bien qu'on ne sache pas si le locuteur est à même de l'identifier (indéfini spécifique) ;

7) La particule nanka d'évaluation négative apparaît dans la plupart des cas dans un contexte où un item à polarité négative est légitimé (ex. dans des phrases négatives, conditionnelles, etc. $)^{19}$. Autrement dit, elle est nécessairement interprétée sous la portée de son légitimateur (indéfini non spécifique).

La particule nanka, qui est à l'origine un SN apposé, donne par ailleurs naissance à un emploi dérivé ; elle peut non seulement signaler la focalisation d'un SN comme en [26]a, mais aussi une suite adverbiale $<\mathrm{SN}+\mathrm{CAS}$ (ABLATIF) > comme en [26]b. Martin (1987) et Teramura (1991), entre autres, observent que la séquence $<\mathrm{SN}+n a n k a+\mathrm{ABL}>$, comme en [26]a, reste ambiguë entre exemplification et évaluation négative, tandis que la séquence $<\mathrm{SN}+\mathrm{ABL}+$ nanka $>$, comme en [23]b, n'admet que la seconde lecture :

[26]a Taro nanka- kara tegami-o morawa-nakat-ta. Taro NANKA Abl lettre-Acc recevoir-Neg-Pas

[i] 'Je n'ai pas reçu de lettre de Taro, entre autres.' (exemplification)

[ii] 'Je n'ai pas reçu de lettre d'un type comme Taro!' (évaluation négative)

[26]b Taro-kara nanka tegami-o morawa-nakat-ta. (adapté de Martin 1987 : 162] Taro-Abl NANKA lettre-Acc recevoir-Neg-Pas

[i] "Je n'ai pas reçu de lettre de Taro, entre autres.' (exemplification)

[ii] 'Je n'ai pas reçu de lettre d'un type comme Taro!' (évaluation négative)

Nominal apposé au début, la particule nanka qui suit la marque casuelle en [26]b obtient un statut adverbial. Le contraste entre [26]a et [26]b indique que l'adverbial nanka n'exprime que l'évaluation négative qui correspond, comme on l'a vu plus haut, à un item à polarité négative, et donc à l'indéfini non spécifique.

Les correspondances formelle et interprétative de la particule de focus nanka sont récapitulées en [27] sous forme d'échelle d'indéfinitude :

[27] réanalyse de la particule de focus nanka

\begin{tabular}{|ccc|}
\hline indéfini spécifique & indéfini non spécifique \\
\hline $\mathrm{SN}[-y a]-$ nanka ${ }^{\text {nominal apposé }}+$ Cas & $\rightarrow$ & SN-Cas+nanka ${ }^{\text {adverbe }}$ \\
[exemplification/évaluation négative] & [*exemplification/évaluation négative] \\
[26]a & [26]b \\
\hline
\end{tabular}

19 Dans certains cas, le nanka d'évaluation négative est légitimé dans une phrase affirmative. Ainsi, [19\}a permet cette lecture, qui consiste à indiquer que le focus Taro est un individu inattendu et inapproprié pour l'événement 'venir'. Kaneko (2006) soutient que la dérivation de la lecture d'évaluation négative dans un tel cas met également en oeuvre la négation impliquée pragmatiquement par le Principe de Quantité de Grice, de la même manière que les questions rhétoriques. 
La distribution de la particule nanka rappelle celle du dare-ka d'indéfinitude récapitulée en [13], mais manifeste une différence importante : en [13], la réanalyse va de l'indéfini non-spécifique à l'indéfini spécifique, tandis que la direction est inversée en [27]. Dans la section 4, je montrerai que les optiques diachronique et typologique appuient l'analyse en [27].

\section{Structure interne complexe et grammaticalisation}

Dans cette section, j'exposerai d'abord, suivant les études diachroniques, l'hypothèse que dare-ka et nani-ka ont à l'origine une structure interne complexe analogue à celle d'une phrase interrogative enchâssée (§ 4.1). Je proposerai ensuite, dans une optique typologique, et contrairement à Mikami, l'hypothèse que dare-ka et nani-ka ont subi une grammaticalisation dans deux directions opposées (§ 4.2).

\subsection{Optique diachronique}

La linguistique traditionnelle japonaise distingue couramment deux types d'interrogation :

toi 'question', qui consiste à demander à un interlocuteur de remplir une valeur [de vérité ou d'une variable] encore indéterminée ;

utagai 'doute', qui consiste à s'interroger de manière monologique en laissant en suspens la valeur de vérité ou la valeur d'une variable. Les deux types d'interrogation peuvent être respectivement paraphrasés en français par je vous demande $Q$ et je me demande $Q$ / je ne sais pas $Q$ [où $Q=$ question. Il est par ailleurs largement admis qu'en ancien japonais du VIII ${ }^{\text {ème }}$ siècle, «[la particule] $K A$ est spécialisée dans l'expression du doute, de l'incertitude, et (une autre particule) $Y A$, dans celle d'une question, d'une demande» (TambaMecz 1984 : 223) ; dans le japonais moderne, qui a perdu la particule $y a, k a$ a étendu sa distribution pour pouvoir exprimer également la question. Mikami (1972) observe toutefois que même en japonais moderne, dans une situation nécessairement dialogique, par exemple quand on exige d'un interlocuteur qu'il donne son identité, on recourt plus naturellement à la forme sans $k a$, comme en [28]a, qu'à la forme avec $k a$, comme en [28]b :

[28] [Le locuteur découvre un inconnu dans son appartement]

[28]a dare-da?

QUI-Cop

'Qui êtes-vous ?'

[28]b \#dare-ka?

QUI-KA

\# 'Je me demande qui vous êtes.'

Sakakura (1960) fait de plus remarquer que le caractère de doute subsiste plus fortement dans le $k a$ d'indéfinitude que dans le $k a$ d'interrogation, et cite un exemple $\mathrm{du}$ XIX ${ }^{\text {ème }}$ siècle, où la copule s'intercale entre le pronom indéterminé dare et la particule $k a$ :

[29] kii- ta yoona na da ga, dare-de- ka at- ta. entendre-Pas-semble-nom-Cop Adversatif, QUI-Cop-KA Cop-Pas 
«C'est un nom qu'il me semble avoir entendu prononcer, mais c'était celui de je ne sais qui [mais ce nom, je me demande à qui il était ]. » (Hizakurige, début XIX ${ }^{\text {ème }}$, cité par Sakakura $1960: 87$ )

Ces observations diachroniques invitent à supposer que dare-ka a à l'origine une structure complexe incluant une copule comme en [30]b, où la particule $k a$ occupe la tête de la phrase enchâssée (CP). Dans cette optique, la phrase interrogative en [30]a et la phrase incluant $<$ dare[+COPULE] $+k a>$ en [30]b sont respectivement mises en parallèle avec [31]a, dont la proposition principale je me demande ou je ne sais pas explicite la nature monologique, et avec [31]b qui inclut le pronom indéfini complexe je ne sais $q u i^{20}$ :

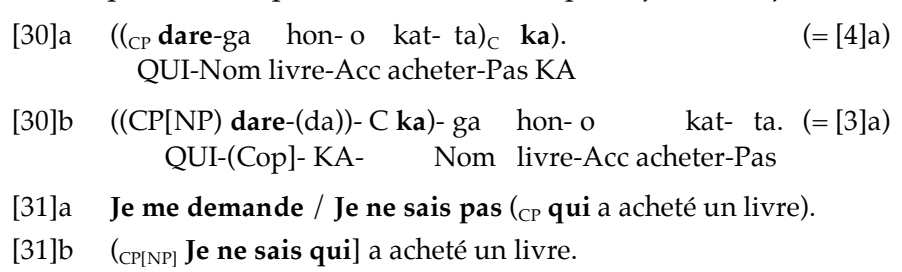

$\mathrm{Au}$ terme de la grammaticalisation, de même que l'expression je ne sais qui en [31]b est réanalysée comme un $\mathrm{SN}$, le $\mathrm{CP}<$ dare+COP+ka $>$ en [30]b est réanalysé, avec l'omission de la partie correspondant à je ne sais, comme un $\mathrm{SN}$ où les deux composants dare et $k a$ sont fortement intégrés. Corrélativement, le complémenteur d'interrogation (plus précisément de doute) $k a$, qui est externe au $\mathrm{SN}$, est réanalysé comme un clitique interne au SN.

Deux faits viennent à l'appui du rapprochement entre dare-ka et la phrase interrogative indirecte. D'abord, Eguchi (1998) signale que la même restriction de cas s'impose à tous les deux : comme on l'a vu dans la $\S 3.1$, le nominatif et l'accusatif sont omissible après dare-ka même dans un registre écrit formel, comme en [32]a, tandis que les autres marques casuelles, comme le datif en [32b], ne peuvent pas être omises. De même, avec la phrase interrogative indirecte, l'accusatif est omissible en [33]a, ce qui n'est pas le cas pour le datif en [33]b :

[32]a dare-ka-(o) syootaisuru. QUI-KA-Acc inviter 'On invite quelqu'un'

[32]b dare-ka-*(ni]) denwasuru. QUI-KA-Dat téléphoner 'On téléphone à quelqu'un'

20 Dans l'optique de Hagstrom (1998) (voir § 2.3), il est possible de supposer que la particule $k a$ est à l'origine adjacente à dare et que la tête de CP est dotée d'un trait interrogatif $\mathrm{Q}$, comme en $(\mathrm{I} a, \mathrm{~b})$ :

(I)a. [[ [

QUI-KA-Nom livre-Acc acheter-Pas

'\{Je me demande / Je ne sais pas\} qui a acheté un livre.'

(I)b. [[ [

QUI-KA-(Cop) Nom-livre-Acc acheter-Pas

'Je ne sais qui a acheté un livre.' 
[33]a (dare-o syootaisuru)-ka-(o) kimeru. (QUI-Acc inviter) -KA- (Acc) 'On décide qui on invite'

[33]b (dare-o syootaisuru)-ka *(ni) kaisya- no mirai-ga kakatteiru. (QUI-Acc inviter)-KA-[Acc] entreprise-Gen futur-Nom dépendre 'Le futur de l'entreprise dépend \{de qui on invite\}.'

Eguchi observe, en outre, que cette restriction de cas disparaît lorsque dare-ka et la phrase interrogative indirecte sont suivis d'un SN, comme en [34] et [35] :

[34] dare-ka tomodati-ni denwasuru.

QUI-KA ami- Dat téléphoner

'On téléphone à un ami quelconque'

[35] (dare-ni ikura kane- o watasu) -ka seejikenkin- no sikata(QUI-Dat combien argent-Acc passer)-KA contribution financière-Gen façonni kaisya- no mirai-ga kakatteiru. [Eguchi 1998 : 9]

Dat entreprise-Gen futur-Nom dépendre

'Le futur de l'entreprise dépend du monde de contribution financière, à savoir, à qui on donne de l'argent et combien'

Ensuite, en japonais contemporain, il est possible de faire suivre dare-ka de l'expression sira, comme l'illustre [36]a. Selon Nihon-kokugo-daijiten (2003 : 622), cette expression est une forme elliptique de sira- $n$ 'je ne sais pas'. Il est raisonnable de supposer que la partie sira est omise dans la plupart des cas au cours de la grammaticalisation. Qui plus est, sira peut aussi être utilisé dans les phrases interrogatives, notamment par les femmes, avec une nuance de modestie, suggérant qu'il s'agit d'un doute plutôt que d'une vraie question, comme en [36]b :

[36]a koko-de-wa itumo dare-ka-sira- ga doko-ka- sira- o Ici- Loc-Top toujours QUI-KA-je ne sais pas-Nom OÜ-KA je ne sais pas-Acc sooji- si- teiru-no da. (K. Ekuni, Garakuta) ménage-faire-Prog-Comp-Cop

(i) 'Ici, je ne sais qui fait toujours du ménage je ne sais où.'

(ii) 'Ici, quelqu'un fait toujours du ménage quelque part.'

[36]b dare- ka ko-nai ka- sira (Y. Kawabata, cité par Nihon-kokugo-daijiten) QUI-KA- venir-Neg KA-je ne sais pas

'Je me demande si quelqu'un ne viendra pas.'

La présence de la copule $d a$ et de l'expression sira est également observée dans un autre emploi grammaticalisé de nani-ka : nani-ka sert aussi de particule de discours et signale que l'on « cherche une expression appropriée, en laissant imprécise de quelle entité, quelle propriété ou de quel événement il s'agit, et peut être paraphrasé par «je ne sais pas si c'est bien dit, mais » (Nihon-kokugo-daijiten 2003 : 209) comme en [37] $\mathrm{a}^{21}$. La particule de discours

21 La particule de discours nani-ka peut être rapprochée de like en anglais, illustré par (Ia), qui « sert à exprimer une éventuelle non-équivalence mineure et non-spécifiée entre ce qui est dit et ce qui est pensé 'is used to express a possible unspecified minor nonequivalence of what is said and what is meant' " (Siegel 2002: 39)», et de genre en français, illustré par (IIb) et défini par Fleischman (1998: 38) comme 
nani-ka est souvent contractée comme en [37]b :

[37]a nnan- da-ka nani-ka sira\} sowasowato otituka- nakat-ta. \{QUOI-Cop-KA / QUOI-KA je ne sais pas\} distrait se sentir à l'aise-Neg-Pas 'Je ne sais pas si c'est bien dit, mais je me sentais distrait et mal à l'aise.' (légèrement modifié d'Okutsu 1996 : 164)

[37]b nanka nihon-no mawari-wa kowai kuni darake da na. QUOI-KA Japon-Gen autour-Top dangereux pays plein-Cop-je crois 'Je ne sais pas si c'est bien dit, mais le Japon est entouré de nombreux pays dangereux, je crois' (20 / 8 / 2006 Journal Asahi)

\subsection{Optique typologique}

L'hypothèse d'une stucture interne complexe est confirmée par l'approche typologique. Haspelmath (1997) rapporte que dans beaucoup de langues, les pronoms indéfinis sont dérivés d'une structure propositionnelle, et cite quatre principales sources :

1) type dunno [qui est une transcription de la prononciation familière de l'anglais don't know), cf. français je ne sais qui / quoi ;

2) type want / please, comme cualquiera en espagnol (quiera est la forme subjonctive de querer 'vouloir'] ;

3) type it may be, cf. français qui / quoi que ce soit ;

4) type no matter, cf. français n'importe qui / quoi.

Dans ces quatre types, la grammaticalisation entraîne la contraction phonologique, l'intégration morphologique, la réanalyse syntaxique et / ou l'extension sémantique ${ }^{22}$.

Selon l’hypothèse avancée plus haut (§ 4.1), la source des dare-ka et nani-ka

servant à « dilue(r) les responsabilités du locuteur quant à la vérité ou à la force assertive du propos de l'énoncé »:

(I)a. It is like the winter in Chicago sucks. (cité dans Fleischman $1998: 36$ )

'Ben, l'hiver à Chicago, c'est vraiment l'horreur.' (traduction de Fleischman)

(I)b. Elle a fait une tête, genre tout le monde devrait être à ses pieds. (Yaguello $1998: 18$

22 La source du déterminant français quelque est du type it may be, comme en (Ia) où les deux parties soulignées, l'adjectif quel et le complémenteur que, sont mises à distance ; à l'étape suivante, lorsque la subordonnée est absente comme en (Ib), les deux parties peuvent être adjacentes, ou bien lorsque la subordonnée est présente comme en $\left(\mathrm{Ib}^{\prime}\right)$, le complémenteur que est dédoublé ; à l'étape finale en (Ic), l'adjectif quel et le complémenteur que sont intégrés pour former un mot - un déterminant :

(I)a. quel samblant qu'el en feîst (Foulet 1982:190) (non spécifique) 'quel semblant qu'elle en fasse'

(I)b. a quel que paine (ibid.) 'avec quelque peine que ce soit'

(I) $b^{\prime}$ an quel que leu que vos ailliez (ibid.) (non-spécifique) 'en quelque lieu que vous alliez'

(I)c. quelque peine (ibid. [non spécifique. / spécifique [ $\pm \mathrm{FAM}]$ ]

$\mathrm{Du}$ point de vue sémantique, la combinaison de quel et que en (Ia) et ( $\mathrm{Ib}, \mathrm{b}^{\prime}$ ) n'exprime que l'indéfini non spécifique, alors que le déterminant quelque en (Ic) exprime également l'indéfini spécifique. 
d'indéfinitude est du type dunno (je ne sais) ${ }^{23}$. D'après Haspelmath, ce type n'admet au début que l'interprétation indéfinie spécifique -FAM. Cette restriction est naturellement expliquée du point de vue théorique : 1] dunno signale l'ignorance du locuteur, tandis que l'indéfini spécifique +FAM implique que le référent soit connu, d'où une contradiction sémantique ; 2] étant donné que la non-spécificité implique par définition que personne n'arrive à identifier le référent, le recours au type dunno pour exprimer la nonspécificité revient à faire une redondance informationnelle, en violation du Principe de Quantité de Grice.

Cette restriction est aussi confirmée empiriquement par le pronom indéfini peu grammaticalisé je ne sais quoi en français. Comme le précise Lefeuvre (2006 : 164), « je ne sais quoi spécifique [...] sollicite l'ignorance du locuteur ». Ainsi, en [38]a, bien que placé dans la subordonnée d'un prédicat principal de modalité irréelle, il faut croire, ce pronom indéfini dénote quelque chose qui existe réellement et n'est donc pas interprété sous sa portée comme non spécifique. L'interprétation spécifique +FAM n'est pas permise non plus, comme le montre [38]b :

[38]a Il faut croire que l'amour, la passion, la tendresse, je ne sais quoi encore, se font leur chemin tout seuls (Lefeuvre 2006 : 163).

[38]b J'ai acheté \{quelque chose / \#je ne sais quoi\}. Devine quoi ! (idem.164).

Dans cette optique, l'indéfini spécifique -FAM doit être l'interprétation de base des dare-ka et nani-ka, et l'indéfini spécifique +FAM ainsi que l'indéfini non spécifique, des interprétations dérivées. L'indéfini spécifique +FAM et l'indéfini non spécifique sont typiquement incarnés respectivement par l'emploi pronominal suivi d'une marque casuelle et par l'emploi adverbial. Il s'ensuit que ces deux emplois sont des expressions grammaticalisées dans deux directions opposées, comme le montre [39], ce qui contredit la thèse de la réanalyse unidirectionnelle de Mikami. La distribution de la particule nanka vient confirmer que l'extension sémantique se dirige de la lecture spécifique vers la lecture non spécifique :

[39] Réanalyses du dare-ka d'indéfinitude et de la particule de focus nanka

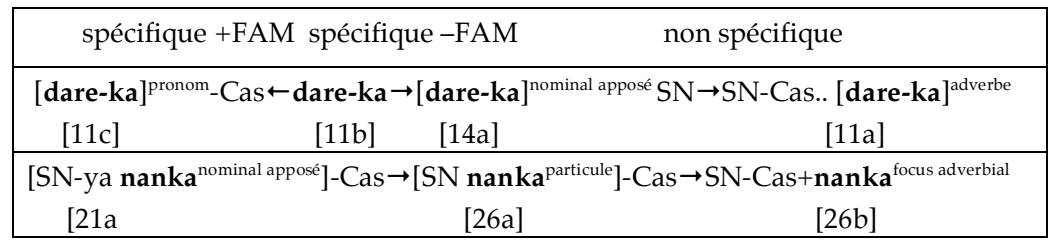

\section{Conclusion}

Dans cette étude, après avoir passé en revue les principaux travaux sur les expressions indéfinies du japonais, j'ai d'abord examiné les propriétés interprétatives de dare-ka et nani-ka dans divers environnements. J'ai ensuite

23 Haspelmath lui-même n'attribue pas la source de dare-ka au type dunno. 
proposé de modifier l'hypothèse de Mikami selon laquelle l'emploi pronominal est dérivé à partir de l'emploi adverbial, en intégrant à la description les propriétés de la particule de focus nanka. J'ai enfin proposé l'hypothèse que dare-ka et nani-ka ont à l'origine une structure interne complexe analogue à celle d'une question enchâssée et suivent diverses voies de grammaticalisation, représentées en [40] par les flèches verticales et horizontales :

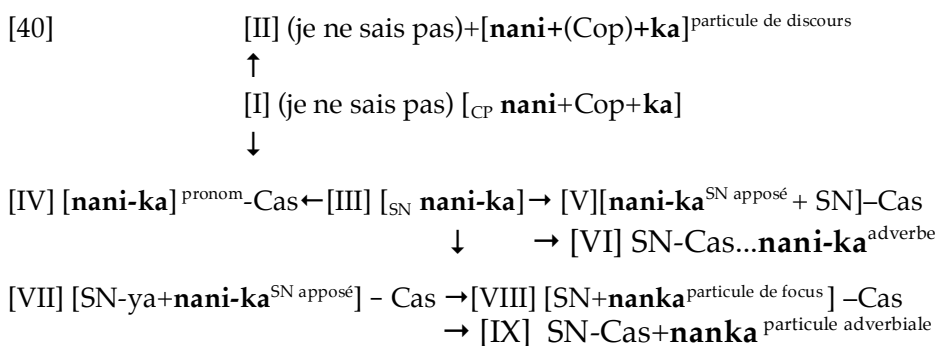

- Le CP nani/dare+copule+ka (cf. [29]) en [I] est grammaticalisé

- soit comme la particule de discours nani-ka (cf. [37]a,b) en [II],

- soit comme le SN nani-ka/dare-ka sans marquage casuel qui permet l'indéfini spécifique -FAM (cf. [11]b), en [III].

- Le SN nani-ka/dare-ka en [III] est à son tour grammaticalisé

- soit comme un pronom qui permet l'indéfini spécifique +FAM, lorsqu'il est suivi d'une marque casuelle (cf. [11]c), en [IV],

- soit comme un $\mathrm{SN}$ apposé lorsqu'il précède $<\mathrm{SN}$-cas $>$ (cf. [14]a), en [V],

— soit comme un SN apposé lorsqu'il suit <SN-ya > (cf. [21a]), en [VII].

- Le SN apposé en [V] est grammaticalisé comme un adverbe lorsqu'il est détaché de $<\mathrm{SN}-\mathrm{CAS}>$ (cf. [11]a), en [VI].

- Le SN apposé en [VII] est grammaticalisé, avec l'omission de ya et au terme d'une contraction phonologique et d'une extension sémantique, sous la forme de la particule de focus nanka (cf. [26]a, en [VIII],

- La particule de focus nanka en [VIII] est de plus grammaticalisée comme un adverbe lorsqu'il est précédé de $<\mathrm{SN}-\mathrm{CAS}>$ (cf. [26]b), en [IX].

Les 'pronoms indéfinis' japonais révèlent ainsi, par leurs diverses voies de grammaticalisation, les relations étroites qu'entretient l'indéfinitude avec l'interrogation et la disjonction ainsi qu'avec la focalisation (entraînant des effets sémantiques tels que l'exemplification, ou l'évaluation négative).

\section{Références}

Blin R. (1994), « Les démonstratifs indéfinis en japonais », Faits de langues 4, L'indéfini : 65-71.

Chang I.-B. \& L. Labrune (1994), « Les « indéfinis » en coréen et en japonais », Faits de langues 4, L'indéfini : 73-80 
Dobrovie-Sorin C. \& Beyssade C. (2004), Définir les indéfinis, CNRS Édition.

Eguchi T. (1998), « Nihongo-no kansetu-gimon-bun-no bonpoo-teki itizuke-nituite (“On the Grammatical Status of Japanese Indirect Questions") ", Kyushu University Annual Report of Linguistics 19 : 5-24.

Enç M. (1991), « The Semantics of Specificity », Linguistic Inquiry 22 : 1-25.

Fleischman S. (1998), « Des jumeaux du discours », La linguistique 34.2 : 31-47.

Foulet L. (1982), Petite syntaxe de l'ancien français, Champion.

Hagstrom P.-A. (1998), Decomposing Questions, Ph.D. dissertation, MIT.

Haspelmath M. (1997), Indefinite Pronouns, Oxford Univ. Presse.

Kadmon N. \& F. Landman (1993), «Any », Linguistics \& Philosophy 16: 353-422.

Kamio A. (1973), "Observations on Japanese Quantifiers », Descriptive and Applied Linguistics 6 (Sophia University, Japan) : 69-92.

Kaneko M. (2006), « La dérivation pragmatique du sens de surprise », communication donnée aux Journées Sémantique E Modélisation, à Bordeaux, le 30 mars 2006.

Kawaguchi J. (1982), « dare-ka + NP », Geibun-Kenkyuu (Keio University) 44 :110-126.

Kratzer A \& J. Shimoyama (2002), «Indeterminate Pronouns: the Views from Japanese », in Otsu, Y. (eds), The Proceedings of the Third Tokyo Conference on Psycholinguistics, Hituzi Pub. Tokyo : $1-25$.

Kuroda S.-Y. (1965), Generative Grammatical Studies in the Japanese Language, Ph.D. dissertation, MIT.

Lefeuvre F. (2006), Quoi de neuf sur quoi : étude morphosyntaxique du mot quoi, Presse. Univ. de Rennes.

Martin S.-E. (1987), A Reference Grammar of Japanese, Charles E. Tuttle Company, Rutland Vermont / Tokyo Japan.

Meillet A. (1958), Linguistique historique et linguistique générale, Honoré Champion.

Mikami A. (1972), Gendai-gohoo-jyosetu (Introduction à l'usage moderne du japonais), Kurosio Pub., Tokyo.

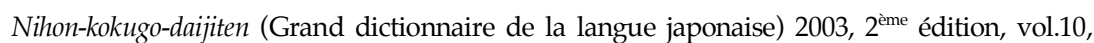
Syoogakukan, Tokyo.

Nishigauchi T. (1990), Quantification in the Theory of Grammar, Kluwer.

Okutsu K. (1996), Syuui Nihon-Bunpoo-Ron (Recueil supplémentaire des articles sur la grammaire japonaise), Hituji Pub. Tokyo.

Rooth M. (1996), « Focus », dans S. Lappin (eds) The Handbook of Contemporary Semantic Theory, Blackwell :271-297.

Sakakura A. (1960), « Bunpoosi-ni tuite : gimonhyoogen-no hensen-o itiree tosite (À propos de l'histoire de la grammaire : un exemple du changement diachronique des expressions interrogatives ", Kokugo to kokubungaku (Langue et littérature japonaises) 37.10:75-88.

Shimoyama J. (2006), «Indeterminate Phrase Quantification in Japanese», Natural Language Semantics $14: 139-173$.

Siegel M. (2002), « Like: The Discourse Particle and Semantics », Journal of Semantics 19: 35-71.

Tamba-Mecz I. (1984), «Expressions interrogatives et particule «ka » en japonais », Linguistica Palatina Colloquia II : 209-242.

Teramura H. (1991), Nihongo-no sintakusu to imi III (Syntaxe et sémantique du japonais III), Kurosio Pub., Tokyo.

Watanabe A. (1992), « Subjacency and S-structure Movement of WH-in-situ », Journal of East 
Asian Linguistics 1 : 255-291.

Watanabe A. (2006), « Functional Projections of Nominals in Japanese: Syntax of Classifiers », Natural Languages and Linguistic Theory 24: 241-306.

Willems D. (1998), « Un petit rien sur quelque chose », dans A. Englebert et al (eds), La ligne claire : de la linguistique à la grammaire, Duculot : 137-145.

Yaguello M. (1998), « Genre, une particule d'un genre nouveau », Petits faits de langue, Seuil: 18-24.

Yatsushiro K. (1996), « The Distribution of mo and ka and its Implications », dans K. Arregi, et. al., MIT Working Papers in Linguistics 33.

Université d'Okayama (Japon) 